\title{
SLIT HOMOGENIZERS FOR EARTH OBSERVATION SPECTROMETERS: overview on performance, present and future designs
}

J.Caron, B.Kruizinga, R.Vink TNO, Optics Department, Delft, the Netherlands 


\section{OUTLINE}

> Introduction to slit homogenizers (SH)

) Two SH designs: mirror-based and lens-based

> Performance at $1^{\text {st }}$ order

> Grating non-uniform illumination, impact on diffraction and $2^{\text {nd }}$ order effects

> Conclusions 


\section{TROPOMI vS SENTINEL-5}

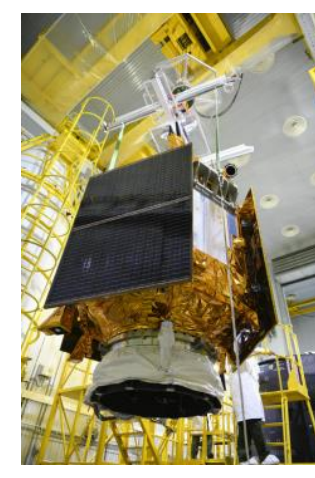

\section{Tropomi / Sentinel-5 P}

- Launched Oct 2017

- Min.lifetime = 7years

- $2600 \mathrm{~km}$ swath

- $7 \mathrm{~km}{ }^{\star} 7 \mathrm{~km}$ sampling

- 4 spectrometers = UV-VIS $(270-500 \mathrm{~nm})+\mathrm{NIR}(675-775$ $\mathrm{nm})+$ SWIR-3 (2305-2385 nm)

- No slit homogenizers

\section{Sentinel-5 / Metop-SG}

- Launch due 2021

- Min.lifetime $=7$ years

- $2600 \mathrm{~km}$ swath

- $7 \mathrm{~km} * 7 \mathrm{~km}$ sampling

- 5 spectrometers = UV-VIS $(270-500 \mathrm{~nm})$ + NIR (685-773nm) + SWIR-1 (15901675nm) + SWIR-3 (2305-2385nm)

- Slit homogenizers in all channels $\rightarrow$ Talk J. Irizar, Sentinel-5

Both instruments have very similar characteristics and strongly overlapping spectral bands. Most likely they will be flying at the same time. 


\section{IMPACT OF NON-UNIFORM SCENES}

Case 1 = uniform

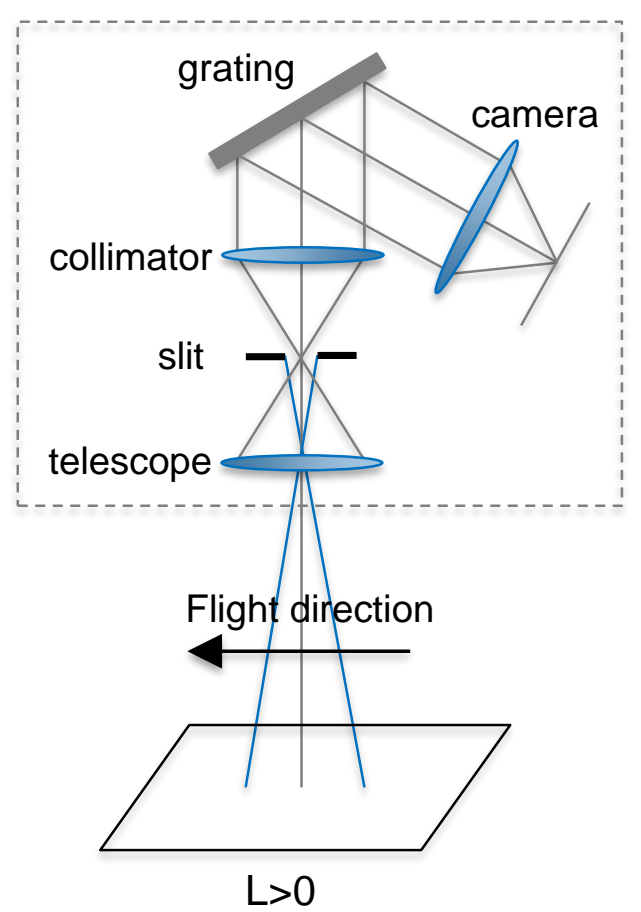

Case 2 = non-uniform

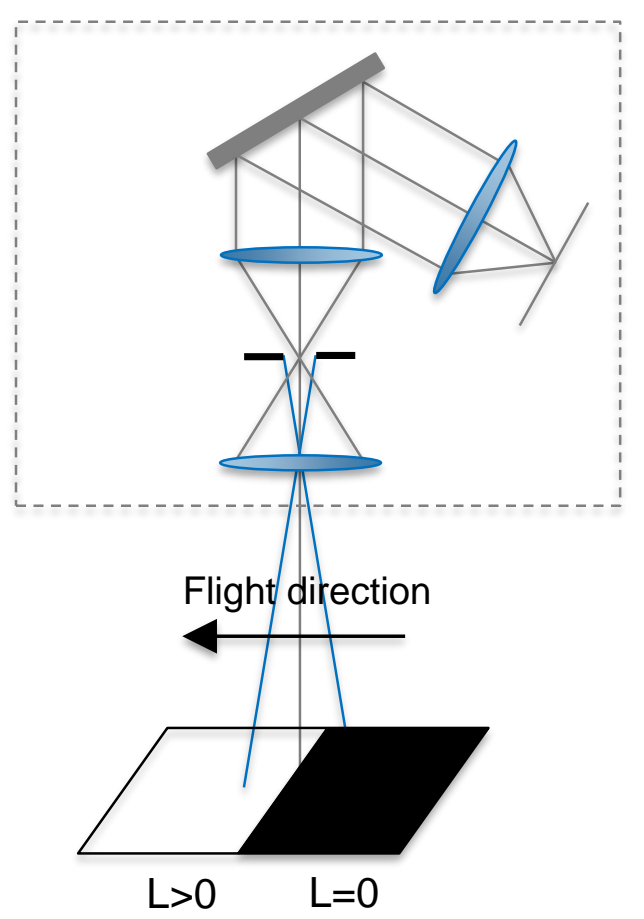

- Two situations with a spectrometer flying over a uniform/non-uniform scene

- same spectrometer measures different ISRF depending on scene
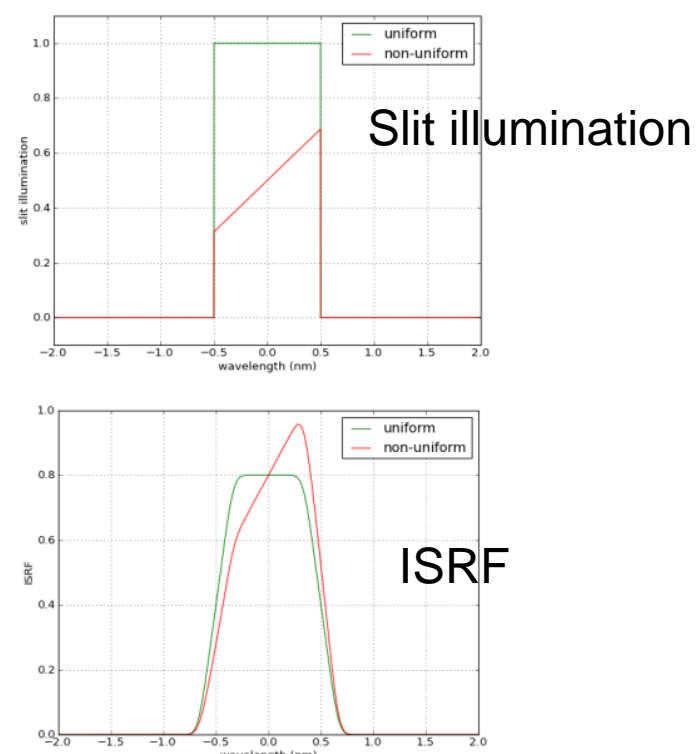


\section{SENTINEL-5 SLIT HOMOGENIZER (MIRROR-BASED)}

1 Sentinel-5 SHs consist of two blocks coated with a highly reflective coating, separated by spacers

1) The beam delivered by the telescope is focused at the $\mathrm{SH}$ entrance and undergoes multiple reflections on the $\mathrm{SH}$ mirrors

1 "Length rule": when the length is an even multiple of
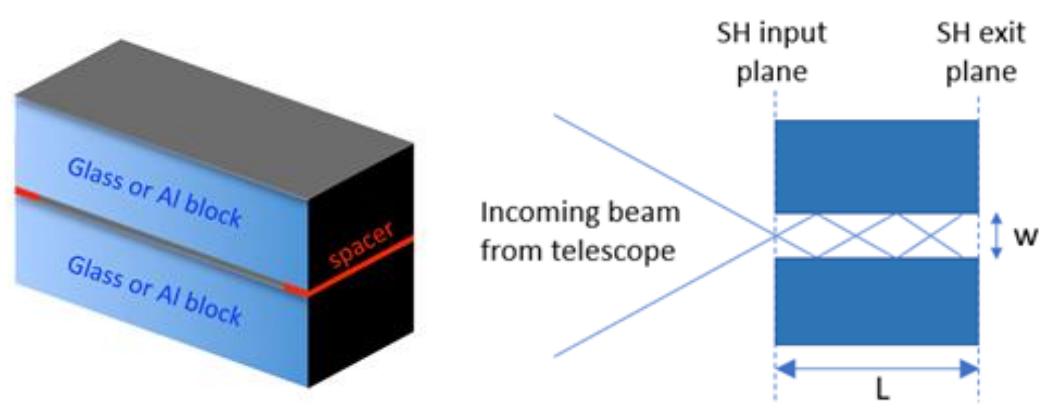
F-number times SH width, a "naïve" geometrical optics reasoning predicts perfect performance of the $\mathrm{SH}$ :

$$
L=2 n w F_{\#} \quad n=1,2,3 \ldots
$$

) This is simplified. In reality the slit intensity distribution at $\mathrm{SH}$ exit is a complex interference pattern

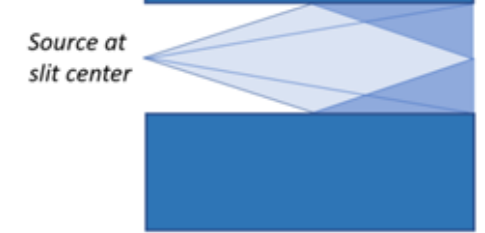




\section{LENS-BASED DESIGN}

) An image of the telescope pupil, that is perfectly homogeneous, is used as a virtual entrance slit for the spectrometer

1 This is performed with a lens rod placed immediately behind the slit. The lens must have only power in ALT. It must be a rod, with any lens-shaped cross-section (cylinder, biconvex, plano-convex, etc)

> Patent Application EP1793278A2

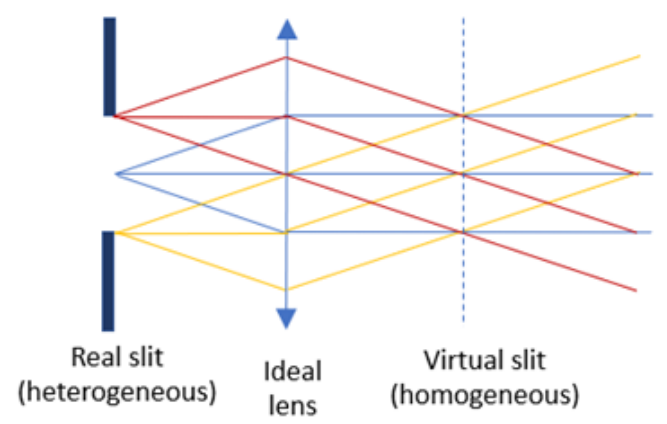

Operating principle

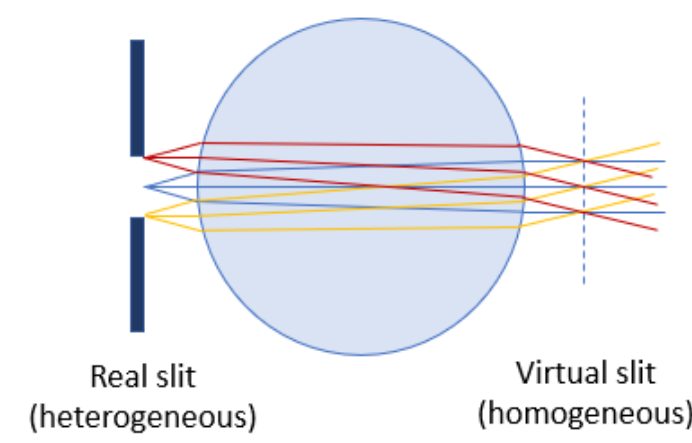

More realistic implementation
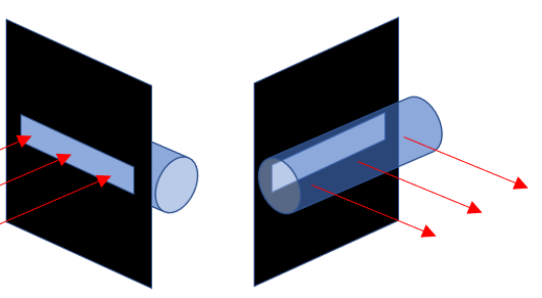

Lens rod behind physical slit 


\section{PERFORMANCE OF MIRROR-BASED SH}

1 Mirror images of a point source at $\mathrm{SH}$ input

> A complex interference pattern is created at the SH output

> It can be predicted with an interference or diffraction model

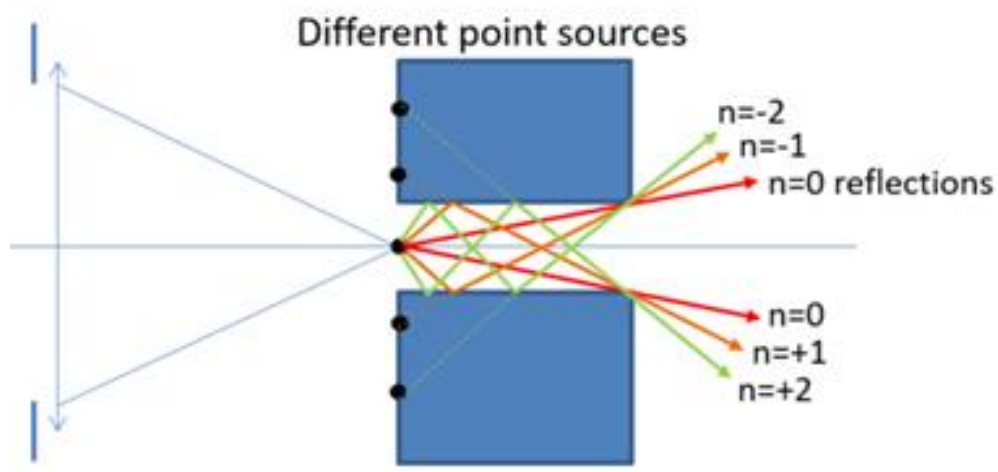

Mirror images at coordinates $x_{n}=n w+(-1)^{n} x_{0}$

7 | ICSO 2018, International Conference on Space Optics, 9-12 October 2018, Chania, Greece

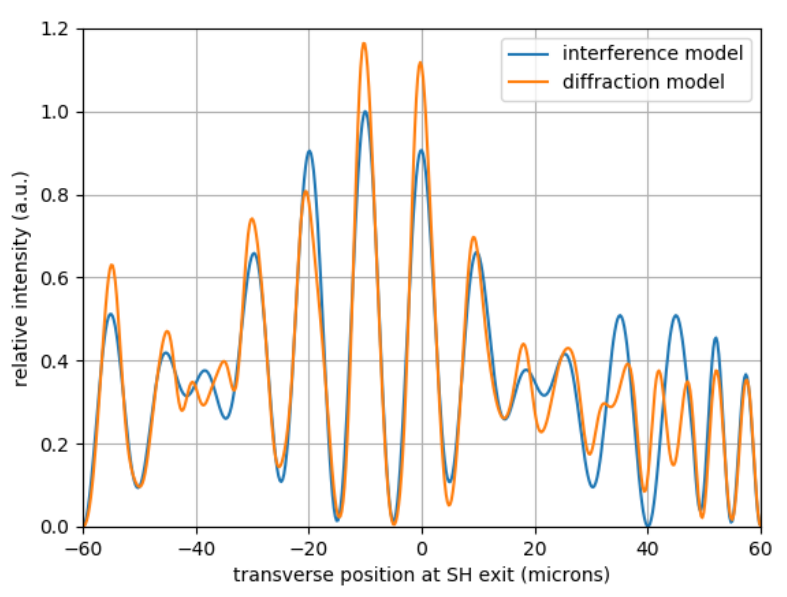

Slit width $w=120 \mu \mathrm{m}$ slit length $L=4.8 \mathrm{~mm}$ reflection $R=-1$ (dephasing $=\pi$ ) F-number $=10$ wavelength $=500 \mathrm{~nm}$ Elementary source at $x_{0}=+48 \mu \mathrm{m}$ 


\section{PERFORMANCE OF LENS-BASED SH}

) The final slit image on the detector is an image of the instrument pupil. It is perfectly homogeneous in all cases and does not depend on the observed nonuniform scene. From this perspective, the lens-based $\mathrm{SH}$ is a perfect homogenizer

1 As we will see, second-order error mechanisms are limiting its performance. The lens-based $\mathrm{SH}$ has a performance that is an order of magnitude better than its mirror counterpart

The virtual slit is an image of the system entrance pupil. It is perfectly homogeneous and used as spectrometer entrance slit

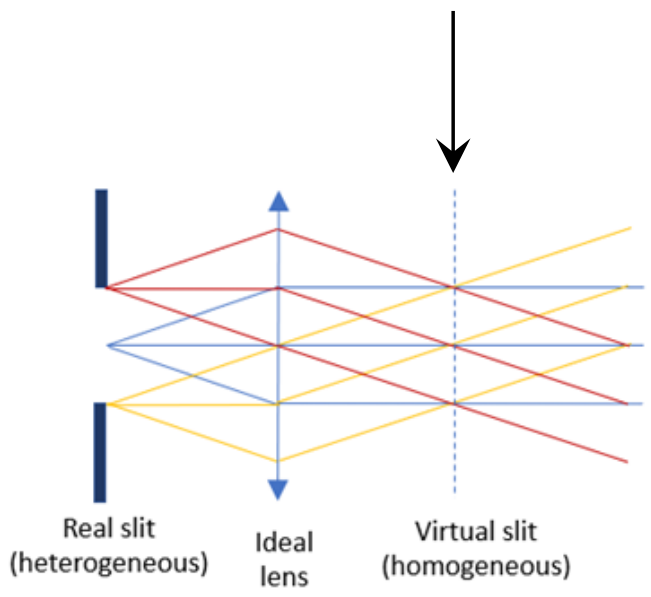




\section{GRATING ILLUMINATION (MIRROR-BASED SH)}

1 The mirror-based SH strongly modifies the pupil (=grating) illumination in the spectrometer

) A simple model predicts an illumination pattern with horizontal "stripes". In reality, it is a more complex diffraction/interference pattern with intensity peaks

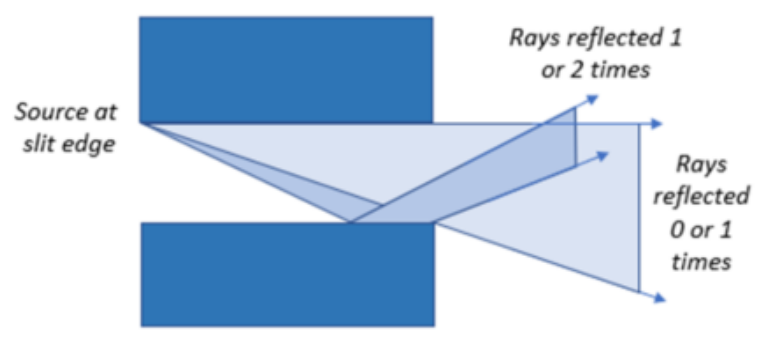

1. The slit homogenizer modifies the angular distribution of intensity

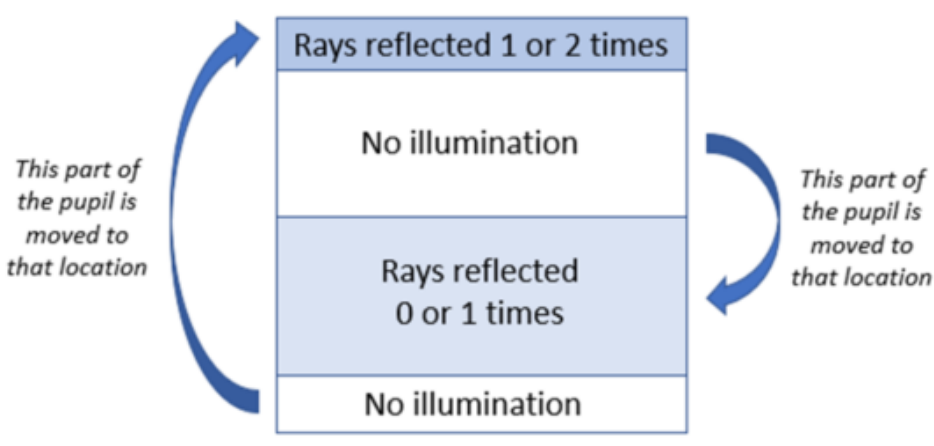

2. The illumination distribution on the exit pupil is modified 


\section{GRATING ILLUMINATION (LENS-BASED SH)}

I In the spectral direction, the lens-based SH exchanges the pupil and image planes

1 A single source point on Earth produces a focused image on the grating (spectral), but illuminates the full grating in the orthogonal direction (spatial)

Remark: In practice the grating is never exactly at the spatial or spectral pupil planes. And these two pupils may not coincide. Additionally, the dispersion generates a spectral image that is much wider than the slit so it creates a new spectral pupil at the grating, but that plays no role in diffraction.

Illumination from 1 coherent point source on Earth

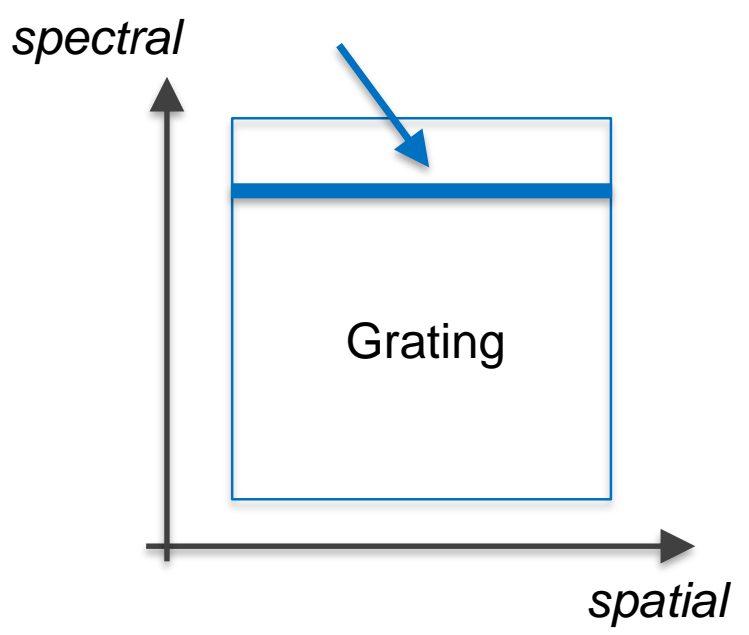




\section{IMPACT ON DIFFRACTION?(1)}

) With both SHs, the grating illumination obtained with one coherent point source is strongly modified. What impact on diffraction and spectral resolution?

1 Common knowledge says that size of grating illumination defines the diffraction pattern. Same reasoning for a mirror. When a plane wave arrives at a mirror, the atoms at the surface act as dipoles and radiate an EM wave in all directions. The emitted wave interferes constructively ONLY around the specular direction. The size of the beam on the mirror defines the angular spread of the reflected light, through diffraction

1 However this picture is only valid when the mirror acts as a physical stop and clips part of the incoming wave. If the mirror does not introduce any clipping, it has NO influence on diffraction
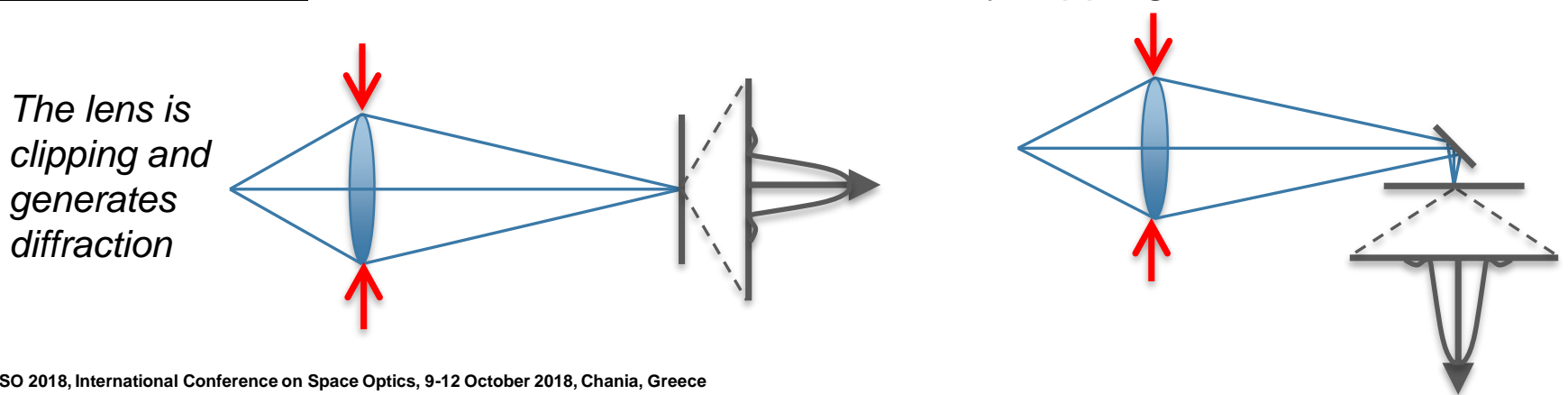

The small mirror does not influence diffraction from the lens. It reflects exactly all the diffracting field. 


\section{IMPACT ON DIFFRACTION ? (2)}

$\checkmark$ In the frame of scalar diffraction theory, the incident field amplitude is multiplied by the grating profile. The grating profile is a convolution between the elementary pattern $G_{\text {pattern }}(x)$ and a Dirac comb.

$$
\boldsymbol{E}_{\text {diff }}(\boldsymbol{x})=\boldsymbol{E}_{\text {incident }}(\boldsymbol{x}) \cdot\left[\boldsymbol{G}_{\text {pattern }}(\boldsymbol{x}) \otimes \sum_{i} \boldsymbol{\delta}\left(\boldsymbol{x}-\boldsymbol{x}_{\boldsymbol{i}}\right)\right]
$$

1 The far-field diffracted field is then obtained by Fourier transform:

$$
\widetilde{\boldsymbol{E}}_{\text {diff }}(\boldsymbol{\theta})=\widetilde{\boldsymbol{E}}_{\text {incident }}(\boldsymbol{\theta}) \otimes\left[\widetilde{\boldsymbol{G}}_{\text {pattern }}(\boldsymbol{\theta}) \sum_{i} \boldsymbol{\delta}\left(\boldsymbol{\theta}-\boldsymbol{\theta}_{i}\right)\right]
$$

1 We see that the grating acts as a convolution kernel that is a sum of Dirac functions (pointing towards the direction of diffraction orders), modulated by the Fourier transform of the grating pattern (grating efficiency). It shows that the grating does not influence the diffraction behavior of the incident beam. 


\section{ADDITIONAL REMARKS}

1 If now some clipping is introduced, the far-field diffraction gets modified so the previous result is no longer true. Then the footprint size (actually grating size) defines diffraction:

$$
\begin{aligned}
& \boldsymbol{E}_{\text {diff }}(\boldsymbol{x})=\boldsymbol{E}_{\text {incident }}(\boldsymbol{x}) \cdot\left[\boldsymbol{G}_{\text {pattern }}(\boldsymbol{x}) \otimes \sum_{i} \boldsymbol{\delta}\left(\boldsymbol{x}-\boldsymbol{x}_{\boldsymbol{i}}\right)\right] . \boldsymbol{G}_{\text {clipping }}(\boldsymbol{x}) \\
& \widetilde{\boldsymbol{E}}_{\text {diff }}(\boldsymbol{\theta})=\widetilde{\boldsymbol{E}}_{\text {incident }}(\theta) \otimes\left[\widetilde{G}_{\text {pattern }}(\theta) \sum_{i} \boldsymbol{\delta}\left(\boldsymbol{\theta}-\boldsymbol{\theta}_{i}\right)\right] \otimes \widetilde{G}_{\text {clipping }}(\theta)
\end{aligned}
$$

I If the groove separation is comparable to the beam footprint on the grating:

$>$ then the grating has a very poor dispersion such that the angular distance between two orders is comparable to the far-field diffraction extent of the incoming beam. As a result the diffracted orders start to overlap and interfere.

I In practice the field angles (in ALT) at the grating are very small, they correspond to the field extent of the slit. The beam arrives at the grating with a large F-number, so the diffraction pattern is relatively large. 


\section{DIFFRACTION IN SPECTROMETERS}

> To predict correctly diffraction effects in a spectrometer, an end-to-end diffraction model is needed.

> Such diffraction modelling can be done with commercial software packages using beam propagation based on gaussian beamlets decomposition (e.g. Fred, CodeV)

> The diffraction behavior of a spectrometer was also investigated with an analytical model by R.Berlich "Radiometric assessment method for diffraction effects in hyperspectral imagers ...", ICSO 2014.

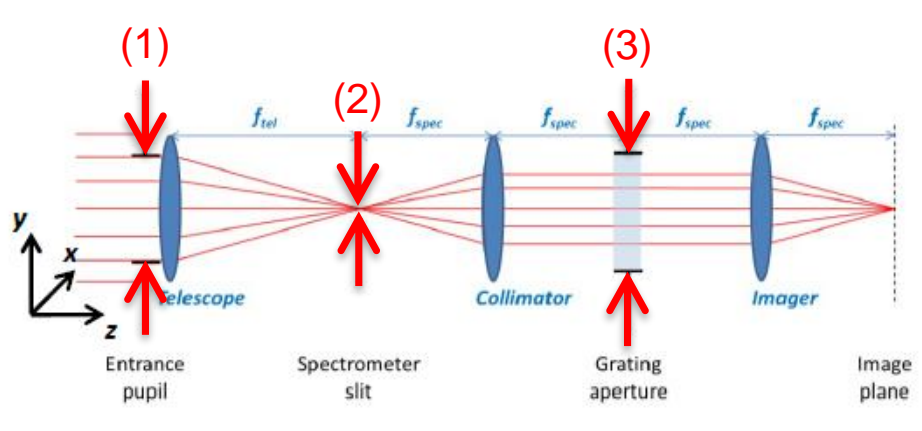

The diffraction pattern on the detector depends on the 3 stops that potentially clip the incoming beam:

(1) the telescope pupil

(2) the physical slit

(3) the spectrometer pupil 


\section{SPECTROMETER DIFFRACTION MODEL}

\section{Summary of the diffraction behavior of a spectrometer (after R.Berlich)}

1. The telescope pupil creates a standard diffraction pattern in the slit plane

2. The physical slit clips the wings of this diffraction pattern. Due to the clipping, a small amount of light is diffracted outside of the nominal range of angles that corresponds to the system F-number

3. To catch the diffracted rays, the spectrometer pupil must be slightly oversized, otherwise it clips the beam again and generate new diffraction.

If the spectrometer pupil is large enough, all diffracted rays are re-imaged and the final image on detector is an exact replica of the clipped diffraction pattern observed just after the slit, with a certain magnification. Diffraction wings at the final image are an order of magnitude lower than what a simple diffraction model predicts before entrance

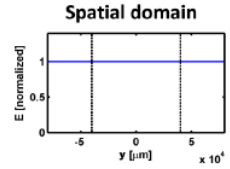

after entrance
pupil

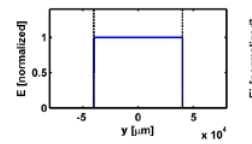

before spectrometer
sitit
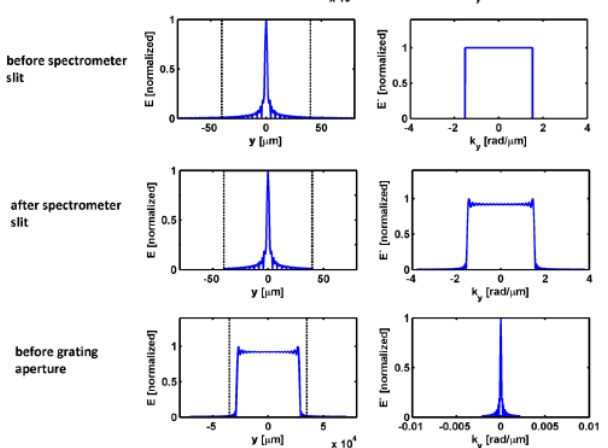

after grating
aperture
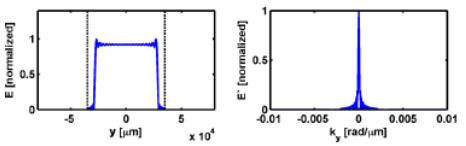

image plane
Angular domain
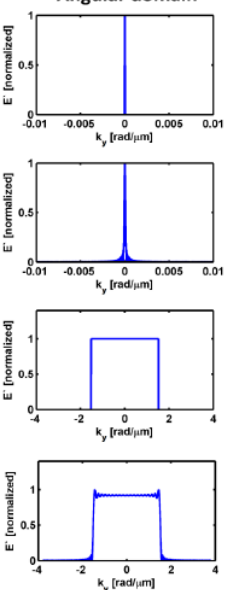

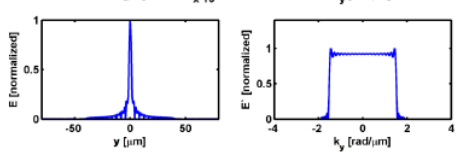




\section{IMPACT OF GRATING NON-UNIFORM ILLUMINATION}

> Previous results are also valid for a system with slit homogenizer (mirror- or lens-based) and suggest that non-uniform grating illumination has no direct impact on diffraction

> On the other hand, a non-uniform grating illumination will influence the ISRF through other mechanisms:

1. Transmittance variations over the spectrometer pupil

2. Aberrations variations over the spectrometer pupil

> A variable non-uniform scene will create radiometric (1) or imaging (2) variations in the final slit image, and therefore cause a small ISRF distortion. These effects have been little studied, new models must be developed. 


\section{CONCLUSIONS}

> Extensive review of the performance of the mirror-based slit homogenizer. In the paper we give analytical expressions to compute the interference patterns, as well as a test case for comparing models.

> New slit homogenizer design, based on a lens rod that performs pupil imaging:

$>$ performance an order of magnitude better than the mirrorbased $\mathrm{SH}$

> can be implemented based on standard optical components

$>$ greater flexibility (no telecentricity required, can be used with any pupil shape) that makes it attractive for a wide range of

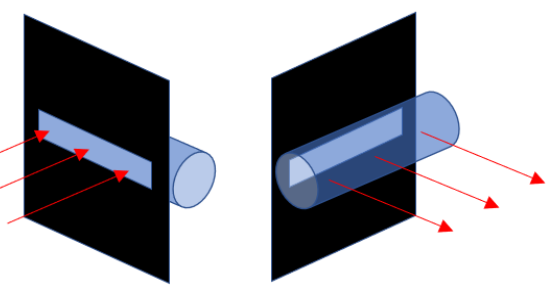
applications 


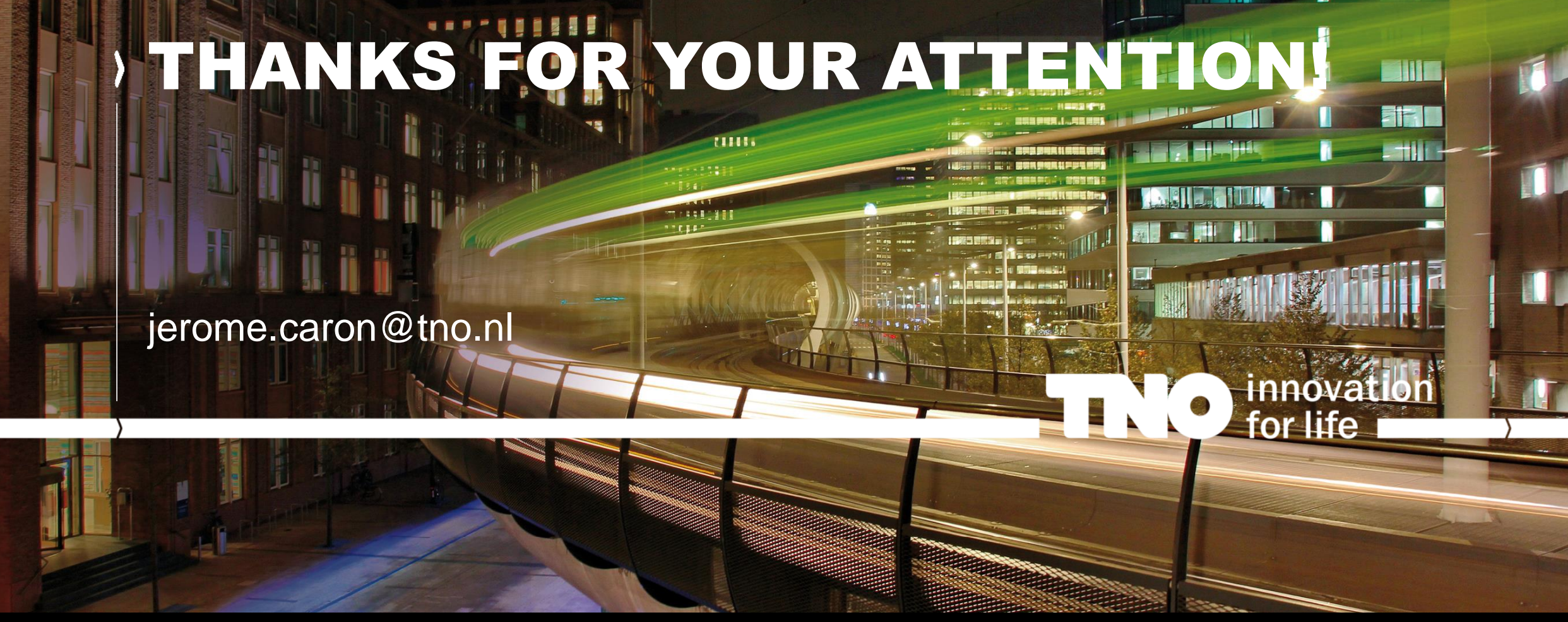

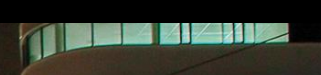
- 


\section{SIZING OF LENS-BASED SLIT HOMOGENIZER}

The SH performs an exchange between pupil and image planes

> The physical slit becomes the pupil $\rightarrow$ its distance to $\mathrm{SH}$ lens defines the pupil position in the collimator

> The telescope exit pupil becomes an image plane $\rightarrow$ its distance to $\mathrm{SH}$ lens defines the virtual slit image position

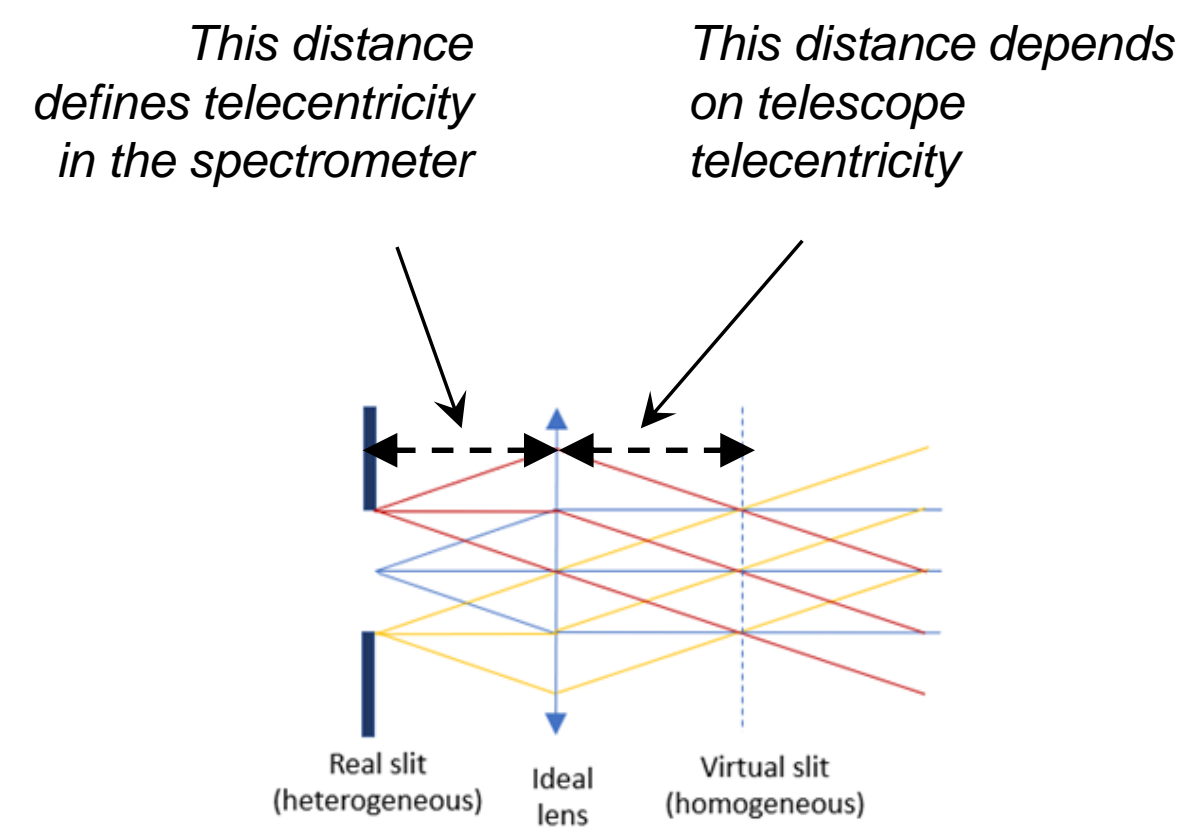

This distance This distance depends on telescope

telecentricity 


\section{NECESSITY OF AN ASTIGMATIC INSTRUMENT}

1 Both slit homogenizers need to preserve image information in ACT + they require a certain length for homogenization

1 Therefore, an astigmatic optical system is required

- In ALT, the telescope image plane coincides with SH input, the collimator object plane with SH output

- In ACT, the telescope and collimator share a common focal plane that can be located anywhere

Example: S5 slit homogenizer with astigmatic telescope

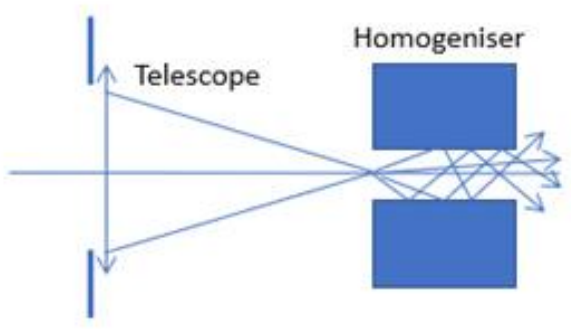

Across slit (homogenising)

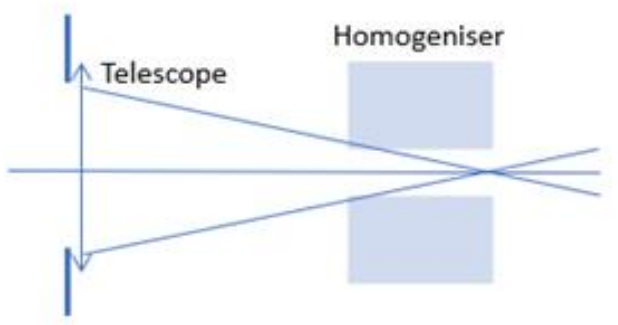

Along slit (image information preserved) 


\section{TRANSFER MATRIX}

1 The interference pattern can be computed at discrete positions at $\mathrm{SH}$ input and output. The resulting intensity map is called a "transfer matrix"

1 The transfer matrix $\mathrm{M}$ allows to compute $\mathrm{SH}$ response $S\left(x^{\prime}\right)$ to any stimulus $S(x o)$ at input. The ISRF is obtained by convolution of $S\left(x^{\prime}\right)$ with the spectrometer PSF and the detector pixel

$$
S\left(x^{\prime}\right)=\sum_{\text {all } x_{0}} M\left(x^{\prime}, x_{0}\right) S\left(x_{0}\right)
$$

) Sum of the matrix $\mathrm{M}$ along rows or columns gives:

- SH response to a uniform illumination at input

- SH transmittance as a function of input position
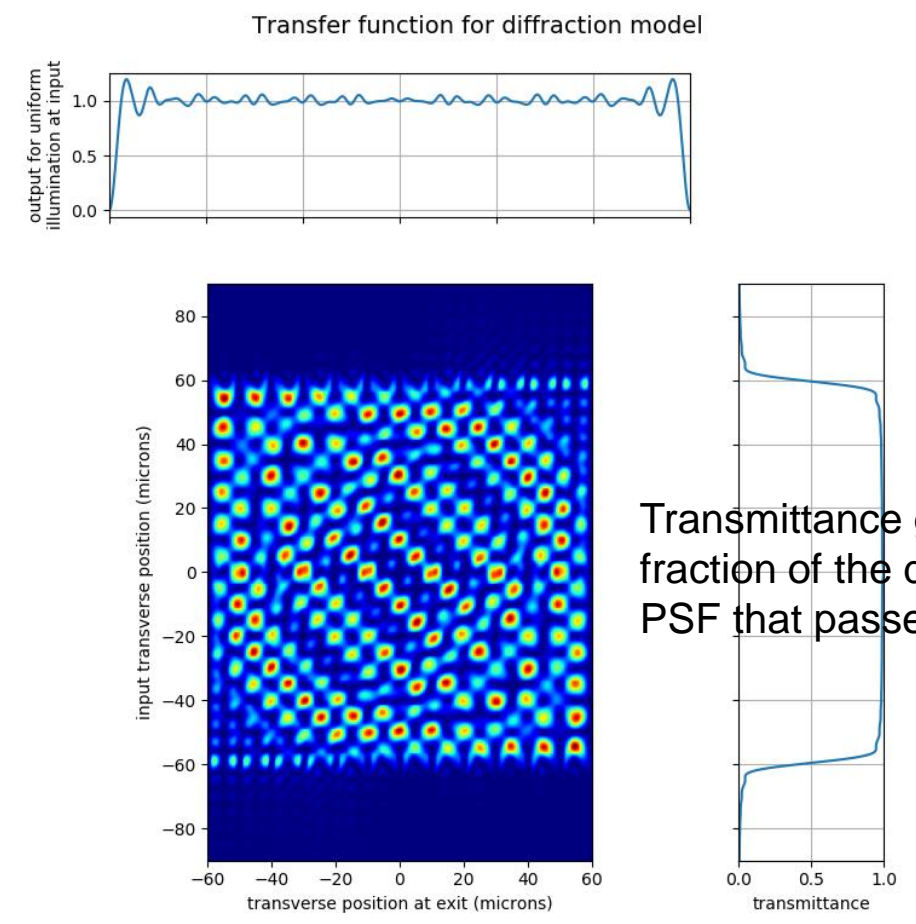